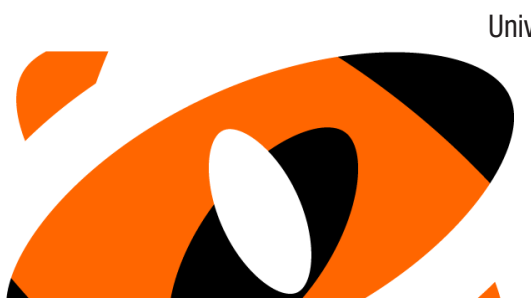

https://artnodes.uoc.edu

\title{
Museos, memoria colectiva e imaginarios narrativos. La comunicación participativa como estrategia para construir relatos no hegemónicos en museos con vocación social
}

\author{
Catalina Gayà Morlà \\ Universidad Autónoma de Barcelona \\ Marta Rizo García \\ Universidad Autónoma de la Ciudad de México \\ Universidad Autónoma de Barcelona
}

Fecha de presentación: octubre de 2021

Fecha de aceptación: enero de 2022

Fecha de publicación: enero de 2022

\section{Cita recomendada}

Rizo García, Marta, Gayà Morlà, Catalina. 2022. «Museos, memoria colectiva e imaginarios narrativos. La comunicación participativa como estrategia para construir relatos no hegemónicos en museos con vocación social». Artnodes, no. 29. UOC. [Fecha de consulta: dd/mm/aa]. https://doi. org/10.7238/artnodes.v0i29.393014
(c)
Los textos publicados en esta revista están sujetos -si no se indica lo contrario- a una licencia
de Reconocimiento 4.0 Internacional de Creative Commons. La licencia completa se puede consultar en https://creativecommons. org/licenses/by/4.0/deed.es

\section{Resumen}

El ensayo plantea comprender los museos como agentes con capacidad de impulsar estrategias de comunicación participativa que permitan la interacción con las comunidades, con la finalidad de que estas participen en la construcción de los relatos que las instituciones museísticas hacen sobre ellas. Partimos de una premisa clara: actualmente, los museos con vocación social se enfrentan a una crisis de participación y de relato. Para enfrentar estas crisis, es apremiante discutir acerca de la participación y las formas de acceso e inclusión de las comunidades en los museos, por un lado, y 


\title{
artnodes
}

https://artnodes.uoc.edu

Museos, memoria colectiva e imaginarios narrativos. La comunicación participativa como estrategia para construir relatos no hegemónicos en museos con vocación social

sobre los imaginarios narrativos que dichas instituciones proponen en sus discursos, por el otro. En un primer momento, se presentan las nociones de comunicación y comunidad de práctica, que entendemos como básicas para la inclusión de los imaginarios colectivos en el relato cultural que proponen los museos. En un segundo momento, se plantea la comunicación participativa como propuesta teórico-metodológica que hace posible la vinculación entre relatos y memorias colectivas. Para ilustrar empíricamente la propuesta, recuperamos algunas conclusiones del proceso de comunicación participativa impulsado por el Museo Marítimo de Mallorca durante 2018 y 2019. El ensayo cierra con algunas conclusiones de la investigación empírica referida y con una discusión abierta que pretende seguir alimentando el debate en torno a la relación entre museos, memoria y relato.

\section{Palabras clave}

comunicación participativa; imaginarios narrativos; memoria colectiva; museo

\section{Museums, collective memory and narrative imaginaries. Participatory communication as a strategy for constructing non-hegemonic stories in museums with a social vocation}

\begin{abstract}
This article proposes that museums should be understood as agents with the capacity to promote participatory communication strategies. These allow interaction with communities so that they can participate in the construction of the stories about themselves that are produced and exhibited by museums. We begin with a clear premise: currently, museums with a social vocation are facing crises of participation and narrative. To resolve these crises, it is vital to debate participation, inclusion, and the types of access that communities have to museums. It is also crucial to debate the narrative imaginaries proposed in the discourse of these institutions. Firstly, the notions of practical communication and community are introduced. These are understood as the basis for the inclusion of collective imaginaries in the cultural narrative proposed by museums. Secondly, participatory communication is suggested as a theoretical-methodological proposal that facilitates the link between narratives/stories and collective memories. To empirically illustrate the proposal, we revisit some conclusions from the participatory communication process undertaken by the Maritime Museum of Mallorca in 2018 and 2019. The article ends with some conclusions from the aforementioned empirical research, as well as an open debate. The aim is to continue to contribute to the debate surrounding the relationship between museums, memory, and narrative.
\end{abstract}

\section{Keywords}

participatory communication; narrative imaginaries; collective memory; museum 


\section{artnodes}

https://artnodes.uoc.edu

«¿Qué se gana y qué se pierde, cuando los seres humanos dan sentido al mundo contando historias sobre el mismo usando el modo narrativo de construir la realidad?» (Bruner 1997, 149)

\section{Introducción}

Más allá de la discusión en torno a la definición de museo social, nuestra propuesta, hecha desde la comunicación, reflexiona sobre los procesos de comunicación participativa ${ }^{1}$ en museos con vocación social. ${ }^{2}$ Así, sin abordar el debate en torno a museologías contrapuestas -un debate que excede el objetivo de este ensayo-, partimos de la premisa de que los museos con vocación social se enfrentan actualmente a una doble crisis: de participación y de relato (Croizet 2021). En este texto, exponemos que, para enfrentar esta crisis y «repensar los museos en términos de compromiso de servicio público» (Loran Gili 2021, 23), urge iniciar un debate en torno a la participación y las formas de acceso, así como en torno a los imaginarios narrativos que los museos proponen en sus discursos.

La Carta de Roma -aprobada en el año 2020 - se refiere a la cultura como «un taller en el que los ciudadanos puedan imaginar respuestas a nuestros retos comunes» $(2020,1)$. Pese a esa necesidad de imaginar en común nuevas maneras de hacer cultura para fortalecer la democracia, el modelo cultural imperante más que un cuestionamiento del relato -y de los procesos con los que este se construye- exotiza a aquellas comunidades que por origen, clase, sexo-género u otro motivo de distinción han sido históricamente etiquetadas como alteridades. ${ }^{3}$ Además, dicha alteridad, como expone García Canclini (2001), demasiadas veces se folcloriza en un discurso velado que no permite a las personas ser agentes creadores de hibridación. Más bien, el relato reproduce los imaginarios sociales ${ }^{4}$ que justifican la construcción de la etiqueta, muchas veces con base en criterios de exclusión e invisibilización.

Asumir la crítica anterior y abordar las crisis de relato supone iniciar un proceso de reflexión de segundo orden ${ }^{5}$ que lleve a los museos a replantearse -como instituciones culturales creadoras de relato cultural público - cómo participan de la vida comunitaria y cómo dicha parti-
Museos, memoria colectiva e imaginarios narrativos. La comunicación participativa como estrategia para construir relatos no hegemónicos en museos con vocación social

cipación puede ampliar las comprensiones del mundo que proponen. Loran Gili lo expone muy claramente: «Per posar les comunitats i els públics al centre, cal un procés que impliqui grups diversos, que escolti necessitats i integri perspectives» (Loran Gili 2021, 23). Reconociendo esta función, proponemos reflexionar en torno a un escenario en el que los museos con vocación social puedan convertirse en instituciones motor en los procesos de comunicación mediada. Entender estas instituciones como motor de dichos procesos nos pone en la tesitura de repensarlas como agentes con capacidad de imaginar e impulsar mecanismos de escucha activa que transformen el acceso y la interacción con las comunidades, haciéndolas partícipes en la construcción de los relatos que la institución hace sobre ellas 0 que les afectan a ellas.

El caso que se expondrá surge de un proceso participativo que inició el Museo Marítimo de Mallorca (MMM) en el año 2018 en el marco de las Primeras Jornadas del Mar organizadas por la institución. Durante las jornadas, la comunidad marítima cercana al museo reclamó a la institución que se acercara a las comunidades pescadoras para salvaguardar las memorias diversas para las generaciones futuras. La investigación se diseñó desde la comunicación participativa, entendiendo que, antes que nada, el propio museo era investigador e investigado y que el proceso debía cambiar la mirada de los y las trabajadoras, así como los discursos en torno a unas comunidades hasta ese momento excluidas del relato museístico y que ahora reclamaban la actualización de un imaginario que explicaba su mundo, pero del que no se sentían ni partícipes ni reconocidas.

Entendemos que, para explicar el proceso iniciado en Mallorca, primero debemos sustentar una discusión acerca de la participación -en el marco de la propuesta de comunicación participativa- y la inclusión de los imaginarios colectivos en el relato cultural.

\section{Comunicación y comunidad de práctica}

El ser humano, como ser fundamentalmente simbólico, antes incluso que racional, se construye individual y socialmente a través de procesos comunicativos. Es por ello por lo que partimos de la necesidad de vincular la comunicación con la participación, con el fin de plantear

1. Nuestra lectura de la comunicación participativa parte de las aportaciones que se hicieron en Latinoamérica desde los años setenta sobre comunicación para el desarrollo y comunicación para el cambio social. Se trata de una propuesta que, desde la comunicación, interviene en las comunidades y las considera sujetos políticos. Nuestra propuesta es continuista con esta corriente y abunda en los cambios que dichas intervenciones suponen en las narrativas.

2. Si bien la definición de museo social aún está en debate -igual que lo está la de museo-y no existe un consenso en torno a ambas, en este texto nos referimos a museos con vocación social como aquellos que, a través de incorporar la reflexión del presente y sus problemas, tienen como eje transversal de sus prácticas despertar la duda y el espíritu crítico, trabajar con el reconocimiento, y cuyos valores son la justicia social, la igualdad, la inclusión y la sostenibilidad (Barrio Fernández 2017)

3. En este sentido no nos referimos a alteridad, como lo hace Krotz (1994), como aquello que nace del contacto cultural y permanente y que pretende captar el fenómeno de lo humano para comprenderlo y convertirlo en palabra, sino más bien como una etiqueta que podríamos considerar incluso dicotómica, ya que, si bien la presencia supone una inclusión, la forma y el empalabramiento de dicha inclusión ya supone una exclusión en sí misma.

4. «Los imaginarios sociales son aquellos esquemas (mecanismos o dispositivos), construidos socialmente, que nos permiten percibir / aceptar algo como real, explicarlo e intervenir operativamente en lo que en cada sistema social se considere como realidad» (Pintos 1995).

5. Entendemos por reflexividad de segundo orden «cuando un sistema observador toma distancia para observarse a sí mismo y, en un distinto nivel de observación observa los procesos y relaciones del sistema» (Aguado 2003: 279). 


\section{artnodes}

https://artnodes.uoc.edu

la comunicación participativa como propuesta teórico-metodológica que permita generar discursos no hegemónicos de una comunidad de práctica determinada. Discursos, los anteriores, que pueden hacer posible la visibilización de la memoria colectiva, ingrediente principal de los imaginarios narrativos. Partimos de la premisa de que toda actividad simbólica y de construcción de sentido -y toda actividad social- se lleva a cabo a través de mediaciones que implican, de una u otra manera, comunicación.

El ser humano «ubicado en tramas de significación que él mismo ha tejido» (Duch 2019, 109) vive en un estado constante de comunicación (Duch 2002, 35). Es, precisamente, concibiéndose como un ser comunicativo que intenta dar un orden al caos que lo rodea. Por ello, y a través de una praxis de comunicación de la contingencia, acaba generando un orden simbólico. Es decir, el trabajo cultural que implica querer dar sentido -querer construir un medio significativo entre lo ausente y lo presente- es, como exponen García Canclini (1982) 0 Tresserras (1996), lingüístico, narrativo, ergo comunicativo. Geertz lo expone de la siguiente manera al afirmar que la cultura denota «un esquema históricamente transmitido de significaciones representadas en símbolos, un sistema de concepciones heredadas y expresadas en formas simbólicas por medio de las cuales los hombres comunican, perpetúan y desarrollan su conocimiento y sus actitudes ante la vida» (Geertz 1987, 20).

En los procesos de comunicación se comparten contenidos cognoscitivos, información. En este sentido, la comunicación «exige algo que compartir, la voluntad de compartir, alguien con quien hacerlo y las acciones de los que comparten: la expresión y la interpretación» (Moreno Pérez 2008). No podemos negar que la comunicación es fundamental en toda relación social; es el mecanismo que regula y hace posible la interacción entre las personas, y con ella, la existencia de las redes de relaciones sociales que conforman lo que denominamos sociedad.

Las personas establecen relaciones con los demás por medio de interacciones que pueden calificarse como procesos sociales. La interacción puede entenderse como el proceso de organización discursiva entre sujetos que, mediante el lenguaje, actúan afectándose unos a otros, es decir, de forma recíproca. La interacción comunicativa designa, entonces, la trama discursiva que permite la socialización del sujeto por medio de sus actos dinámicos, en tanto que imbrican sentidos en su experiencia de ser sujetos del lenguaje. Por tanto, interactuar es participar en redes de acción comunicativa, es decir, en redes discursivas que posibilitan la aprehensión, comprensión e incorporación del mundo (Rizo 2005).
Museos, memoria colectiva e imaginarios narrativos. La comunicación participativa como estrategia para construir relatos no hegemónicos en museos con vocación social

En una comunidad de práctica, refiriéndonos a esta en el sentido en que lo hace Wenger (1997), como la construcción de un sentido de pertenencia grupal a partir de un proceso de aprendizaje colectivo dentro de un determinado ámbito de interés, la comunicación funge como el mecanismo que regula y hace posible la interacción entre las personas $y$, a la vez, las redes de relaciones sociales que conforman la comunidad. Así, en una comunidad de práctica, la interacción comunicativa permite poner en diálogo y perpetuar (o poner en duda) la identidad ${ }^{6} \mathrm{y}$ el sentido colectivo que, a partir de las relaciones generadas, ha construido y con el que se ha posicionado dicha comunidad. Entendemos identidad colectiva como: «la percepción subjetiva que construyen los miembros de la colectividad sobre los elementos culturales que constituyen la especificidad del grupo, a esos rasgos se les denomina referentes identitarios» (Maldonado y Hernández 2009, 248).

Domínguez García resalta que «los miembros que integran dicha comunidad [una comunidad de práctica] son personas dedicadas a una práctica compartida basada en intercambiar toda una serie de recursos (experiencias historias, formas de pensamiento), a partir de un saber colectivo, generado de manera más o menos autoconsciente» $(2020,14)$.

El papel de la comunicación en esta perpetuación de la doxa y el habitus -en los términos de Bourdieu- de una comunidad de práctica hasta convertirse ambos en discurso nos parece relevante, ya que, por un lado, nos permite entender la negociación entre lo hegemónico y lo no hegemónico en dicho discurso, y por el otro, nos permite revisar relatos hegemónicos y hacer una propuesta en la que la comunicación participativa se convierte en método y metodología ${ }^{7}$ para generar discursos no hegemónicos en torno a dichas comunidades. En este sentido, nuestra propuesta pasa por reflexionar sobre cuál es el papel de los museos con vocación social en su vinculación con las comunidades. La propuesta, además, nos permite revisar las narraciones y la presencia 0 ausencia de las comunidades en estas, así como los relatos (Lynch, 2005) sobre los que se estructuran las narraciones. Recordemos que la capacidad simbólica y comunicativa del ser humano se vehicula de forma compleja a través de la narración que, a su vez, transmite relatos del medio significativo y cultural en el que crece y se interpela por el sentido y por la identidad. En este sentido, si el ser humano es capaz de generar nuevos imaginarios narrativos en torno a su existencia y sus prácticas, y así construir una memoria colectiva, cabe preguntarnos qué rol tienen las instituciones cuyo papel es, entre otros, generar relatos que ayuden a las personas a identificar estructuras asimilables de significación e interpretación de sus identidades y experiencias.

6. Desde los estudios culturales, poscoloniales y decoloniales se ha intentado desestabilizar una noción de identidad excluyente, heteropatriarcal y normativa, repensando las consecuencias de las representaciones y narrativas culturales que mantienen estas relaciones de poder al tiempo que esconden dispositivos de control social, de dominio y de hegemonía de los significados sociales en la sociedad (Hall 1997). Si bien nos parecen imprescindibles las aportaciones de estos enfoques, en este ensayo asumimos un posicionamiento interdisciplinario y multidimensional, pues entendemos que abordar la relación entre comunicación, memoria, narrativa, imaginarios y museos debe tomar en cuenta necesariamente aportes no solo de las ciencias de la comunicación, sino también de la sociología de la cultura, de la museología, de la filosofía y de la lingüística.

7. Abordamos la comunicación participativa como metodología, es decir, como una estrategia general, que se compone de métodos concretos. Como método, debe entenderse como el conjunto de sistemas analíticos que nos permitirán avanzar en nuestro descubrimiento. 


\section{artnodes}

https://artnodes.uoc.edu

\section{Comunicación participativa: estrategias para vincular relato y memoria colectiva}

La comunicación permite a los sujetos participar en prácticas culturales compartidas con fines emancipatorios, es decir, en procesos de intercambio y construcción de significados y sentidos. Entendida así, la comunicación como dispositivo de construcción colectiva de sentidos puede poner en duda los relatos dominantes y generar otros relatos posibles. Permite también generar nuevos modos de construcción de conocimiento sobre determinados fenómenos. Así, aunque toda comunicación es participada, en estas páginas nos interesa poner el énfasis en el concepto de participación como práctica que nos permite, a través del diálogo, reconocer, reconstruir y resignificar la experiencia humana. Proponemos detenernos en las posibilidades que la comunicación nos brinda como acción política de emancipación, ${ }^{8}$ y para ello nos parece sugerente traer a colación un acercamiento al concepto de comunicación participativa (Tufte 2015, Ramiro Beltrán 2005, Gumucio Dagrón 2008) como herramienta teórico-metodológica para la generación de relatos emergentes que cuestionan la hegemonía y proponen nuevos imaginarios narrativos.

En un contexto de revisión de relatos, la comunicación participativa supone implementar un proceso de reflexividad de segundo orden ${ }^{9}$ en las propias instituciones culturales. Dicho proceso les debe permitir, en primer lugar, reconocer la invisibilización de los sujetos o comunidades del relato que proponen y, en segundo lugar, aflorar las violencias y los procesos de dominación por los que han sido marginados de dicho relato. Así mismo, debe ayudar a entender el camino para que sujetos y/o comunidades participen de forma plena en la construcción de los relatos culturales que los pretenden identificar y que lo hagan a partir de modelos de participación no verticales. Así, la comunicación participativa puede ser pensada como acto de interacción horizontal que conlleva a la participación de sujetos diversos -hasta ahora excluidos, invisibilizados, marginalizados- en el establecimiento de nuevos relatos.

De esta manera, la propuesta que hacemos en torno a la comunicación participativa aflora como un artefacto posibilitador de nuevos relatos distintos a los hegemónicos. Emerge, podríamos decir, como una acción incómoda para la hegemonía, ya que en el proceso simbólico de interacción, participación y mediación de sujetos (Martín Barbero 1989) y/o comunidades permite el acceso y la participación en la toma de decisiones de sujetos hasta ahora excluidos, visibiliza una denuncia de los mecanismos de subordinación, opresión y dominación y cuestiona los privilegios a los que dichos mecanismos responden. ${ }^{10}$ Recordemos
Museos, memoria colectiva e imaginarios narrativos. La comunicación participativa como estrategia para construir relatos no hegemónicos en museos con vocación social

que, en una comunidad, el relato posiciona a los sujetos en torno a esta y al imaginario social que estos sujetos comparten y, al mismo tiempo, posibilita la articulación de una narración, que puede ser excluyente u homogeneizadora 0 , por el contrario, equitativa y diversa.

Imaginario, memoria y narración van de la mano. Afirma Mendoza que «la memoria es narrativa en un doble sentido, como relato de progresión de acontecimientos en el hilo del tiempo, y como conformación de una trama (con actores, escenarios y acciones), y de ser verosímil, no verdadero, es aceptado en la medida en que se adecue, 0 acerque, a criterios validados socialmente: existen formas convencionales de cómo narrar 0 dar cuenta de los eventos. Y lo que se narra debe tener sentido. Así, pueden encontrarse muchas narraciones, pero no todas son aceptadas como válidas» $(2004,6)$.

Como las narraciones, los relatos nunca son neutrales ni objetivos, nunca son ingenuos. Como constructores de realidad, tienen una función con respecto a la persona y a la cultura, ya que nos otorgan identidad (Bruner 2003) y, en el proceso intersubjetivo, permiten un proceso dialéctico de reconocimiento. Así, los relatos pueden comprenderse como la producción discursiva para la articulación de sentidos que los miembros de una comunidad construyen en el momento de ser interpelados en torno a diferentes aspectos de la comunidad a la que pertenecen.

Si bien la capacidad narrativa comunitaria -procurada por la comunicación participativa- nos identifica como sujetos en la cultura hegemónica, también es una puerta abierta a la construcción de metarelatos que conforman comunidad, y, por ende, identidades y memorias individuales y colectivas. Mendoza recupera a Halbwachs y Blondel al considerar que la memoria colectiva «es un proceso social de reconstrucción de un pasado vivido y/o significado por un grupo o sociedad, que se contiene en marcos sociales, como el tiempo y el espacio, y como el lenguaje, pero también se sostiene por significados, y éstos se encuentran en la cultura. Si, porque a decir de estos autores la memoria mantendrá aquello que considere significativo, con sentido; no ocurre a la manera de Funes el memorioso, de Borges, que retenía todo lo que veía 0 experimentaba. Ese no es el caso de la memoria, pues en ella se contiene lo que valga la pena guardar, aquello que cobra sentido» (Mendoza 2004, 3).

En un sentido similar, «la memoria es una instancia central en los procesos colectivos, procesos colectivos no simplificados a las dinámicas de relaciones entre sus miembros sino referidos a las producciones subjetivas y dimensiones imaginarias -que son histórico-sociales-, que hacen ser colectivos a los sujetos» (Manero y Soto 2005, 180).

8. Claire Bishop (2012) postula el papel de la política del arte y del papel de espectador. Defiende la necesidad de promover múltiples deconstrucciones como una posibilidad de la política del arte. De este modo, los públicos desarrollan su emancipación no por una situación de aprendizaje único, sino por tratarse de espacios abiertos de lecturas múltiples, de lugares de encuentros y desencuentros, de relaciones y fricciones.

9. Entendemos por proceso de reflexividad de segundo orden «cuando un sistema observador toma distancia para observarse a sí mismo y, en un distinto nivel de observación, observa los procesos y relaciones del sistema» (Aguado 2003, 279). En el ámbito de la investigación social, los procesos de reflexividad de segundo orden emergen cuando la propia investigación genera nuevos modos de percibir y pensar el mundo por parte tanto de los propios investigadores como de los sujetos investigados.

10.Garoian (2002) lo conceptualiza como mediación subversiva, refiriéndose a las diferentes narrativas del museo. 


\section{artnodes}

https://artnodes.uoc.edu

Y los sujetos solo pueden ser colectivos en el marco de procesos de comunicación en los que se encuentran inmersos cotidianamente.

La comunicación participativa puede comprenderse que propicia una comunicación comunitaria, ya que supone un modelo circular que, en el propio proceso, crea comunidad y relato en torno a esta. En este sentido, el relato que emerge de la interacción no solo afecta a los sujetos participantes: ayuda a significar la vida cotidiana más allá del relato hegemónico. Siguiendo con la exposición de Mendoza, entendemos que "puede advertirse que pertenecer a una cultura es encontrarse inmerso en un sinnúmero de relatos interconectados en torno al pasado, aunque no todos ellos establezcan un acuerdo, un consenso. Pueden presentarse los disensos, discontinuidades, desavenencias» (Mendoza 2004, 5).

El relato que emerge del proceso de comunicación participativa se configura como una posibilidad para comprender lo cambiante del entorno, para comprenderse las personas unas a otras, en sociedades diversas y complejas que enfrentan retos ineludibles como la igualdad real o la justicia social y que pone en duda el relato hegemónico y sobre todo el proceso de construcción de este. Es más, dichos retos solo se pueden abordar si hay una verdadera participación de sujetos diversos y si, en el proceso de interacción, se plantea la emergencia de metarrelatos construidos desde agendas temáticas no hegemónicas.

\section{Participación y recuperación de la memoria colectiva de la comunidad marítima del puerto de Sóller: relato de un proyecto de comunicación participativa}

La investigación se enmarca en el proceso comunitario participativo que puso en marcha el Museo Marítimo de Mallorca en el año 2018. Durante las Primeras Jornadas del Mar surge la demanda de la comunidad marítima de que el museo trabaje con comunidades pescadoras de las islas para documentar la pérdida del patrimonio inmaterial de la pesca. Se decide trabajar en el puerto de Sóller, donde el MMM tiene una de sus sedes, el Museu de la Mar. Se diseña un primer acercamiento a los pescadores mayores del puerto, todos ellos retirados, y se les invita a participar en un primer encuentro, a modo de grupo de discusión, en el museo para debatir posibles líneas de trabajo. En este primer acercamiento, aparece que la comunidad de pescadores, pese a que el museo físicamente está ubicado en su barrio, no se siente ni interpelada ni representada por la institución. Es más, los pescadores sienten que el relato que se explica en el museo no solo no los toma en cuenta, incluso que niega su existencia, ya que no se les menciona.

Se contacta con la confradía de pescadores y esta ejerce de puente entre las investigadoras y los pescadores mayores del puerto. Se decide que la búsqueda de testimonios sea abierta, solo que todos ellos tienen que haber trabajado con las artes de pesca tradicionales y haber nacido entre 1929 y 1945.
Museos, memoria colectiva e imaginarios narrativos. La comunicación participativa como estrategia para construir relatos no hegemónicos en museos con vocación social

Se decide diseñar una intervención con las bases metodológicas de la Investigación Acción Participativa (IAP) (Mendia, Luxán etal. 2015). La IAP supone una metodología de investigación y aprendizaje colectivo, basado en la participación activa de los sujetos en todas las fases de creación, lo que nos parece también interesante, ya que supone retos para la propia institución y genera indicadores para enfrentar la crisis de acceso, participación y relato evidentes tras ese primer encuentro. La IAP parte de dos ejes:

a) acercarse a la comunidad pescadora del puerto de Sóller con la intención de que esta se acerque (y se apropie) del museo, y

b) a través de las historias de vida de los diferentes miembros de la comunidad configurar un relato cultural no hegemónico del mar que nos ayuda a entender cómo vivimos y cómo podemos vivir.

Se plantea un paquete técnico que combina entrevistas personalizadas, observación en el barrio de pescadores, historias de vida de miembros de la comunidad pescadora e investigación de imágenes históricas, que los propios sujetos entrevistados aportarán y relatarán en las diferentes sesiones de trabajo. Las entrevistas personalizadas y las historias de vida nos acercan al imaginario colectivo de la comunidad. En este sentido, en las entrevistas desarrollan los indicadores oficio/cambios y en las historias de vida, familia/comunidad/comunidad de práctica. El archivo documental nos sirve para contextualizar la importancia del barrio pesquero en el imaginario social y en la narración colectiva de este. La observación, de nuevo, nos devuelve al barrio y se desarrolla en torno a la expulsión de la comunidad y las consecuencias del turismo.

El universo de entrevistados es de 12 personas, siete hombres y seis mujeres. Los hombres son seis pescadores y un maestro de ribera y las mujeres son cuatro vendedoras de pescado, una esposa de pescador y una hija y hermana de pescador. Se llevan a cabo sesiones de entrevista en el museo y, posteriormente, un trabajo de historias de vida de en sus casas. La observación por el barrio se construye a través de las historias de vida: buscar esos espacios que ellos y ellas empalabran como propios de la comunidad de pesca. Una sesión de observación se hace junto con dos de los pescadores entrevistados. Otra sesión se comparte con dos de las mujeres.

La investigación se alarga 15 meses, de mayo de 2019 a julio de 2020, un año además atravesado por la pandemia.

\section{Algunas conclusiones de la investigación para situar la discusión}

La IAP emerge como una metodología de investigación que visibiliza un relato no hegemónico de la pesca, al mismo tiempo que acciona mecanismos de interacción entre museo y comunidad. En este sentido, la propia comunidad es la que exige la participación en el relato y actividades del museo, más allá de la propia investigación. Es decir, trans- 


\section{artnodes}

https://artnodes.uoc.edu

forma el relato, genera mecanismos para nuevas narrativas (nuevos temas-nuevos actores) y la interacción continuada entre comunidad y museo emerge como necesaria para la existencia de un museo con vocación social, más allá de la investigación e incluso de las narrativas de la pesca.

Al inicio de la investigación, no existe una comunidad pesquera en el puerto de Sóller; existen sujetos individuales relacionados -a veces enfrentados- por vínculos familiares o vecinales que se han dedicado a la pesca artesanal. Al inicio del trabajo de campo emerge la necesidad de purgar otro relato que calificamos como ajeno, pero hecho propio por esta generación de pescadores: el relato del poder, es decir, el de los grandes navegantes que viajaron a Francia a finales del siglo XIX y establecieron un comercio floreciente entre el puerto de Sóller y los puertos del sur de Francia. De hecho, dicho relato ajeno ni siquiera es el de los padres 0 abuelos que navegaban en dichas naves como marinos pobres, sino que es el de los ricos armadores propietarios de las naves con las que sus ancestros viajaban. Ese relato ajeno es el relato que se muestra en el Museo de la Mar y con el que se habían mostrado críticos por no incluirlos a ellos.

La investigación nos permite documentar y conservar un patrimonio inmaterial acumulado en la memoria de los pescadores y vendedoras de pescado del puerto de Sóller y, a través de la participación y de la construcción de relato, los sujetos individuales reconocen la existencia de una comunidad de práctica. El museo, en este sentido, se convierte en la institución propiciadora o promotora de esta comunidad de práctica, ya que en el hecho de poner en valor la práctica de la comunidad y empalabrarla construye un relato que los identifica como comunidad. Los hombres se refieren a los pescadores del puerto sin reconocer, en un principio, la existencia de las mujeres como parte de la comunidad pescadora. Ellas, en cambio, se autoperciben como pieza fundamental de la comunidad de práctica. ${ }^{11}$

Durante la investigación surge una agenda de temas de la que el museo era ajeno: infancias robadas, alcoholismo, resistencias. Es decir, la investigación amplía las temáticas del relato.

Una vez que se ha llevado a cabo la investigación, la comunidad de entrevistados exige que su relato sea parte del museo, más allá de la propia investigación, es decir, como pieza expuesta. Dicha exigencia supone una negociación sobre cómo quiere ser representada la comunidad y cómo quiere y puede representarla el museo. La institución propone que lo sea mediante la museización de un espacio hasta ese momento vacío y se crea la sala de la Remor de la memòria, donde, por medio de un audiovisual y de una intervención artística, las personas con las que se ha trabajado narran ese mundo, el de la pesca artesanal, que está en vías de desaparición del puerto. Sus historias de vida también suponen la narración de los últimos cien años de un territorio: así, la reflexión en torno a la pérdida de la pesca artesanal
Museos, memoria colectiva e imaginarios narrativos. La comunicación participativa como estrategia para construir relatos no hegemónicos en museos con vocación social

deriva en una reflexión sobre la explotación del territorio, la llegada del turismo masivo y cómo afectó a las formas de organización social y del territorio, la adaptación y adopción de tecnologías y costumbres nuevas, muchas veces sin una reflexión necesaria, lo que ha supuesto una crisis de sostenibilidad para el territorio que habitan.

Finalizada la investigación, se deben negociar nuevos escenarios de participación en los que la comunidad siga siendo parte del museo. El hecho de no haber tenido en cuenta estos escenarios durante el proceso de investigación genera malestar y abandono por parte de algunos miembros de la comunidad.

\section{Discusión abierta}

Repensar la crisis de participación y relato de los museos con vocación social desde la comunicación participativa supone inevitablemente plantearse, primero, cómo el museo ha construido un relato hegemónico que nos ha situado -e instalado- en el tiempo y el espacio; nos ha identificado y explicado colectivamente. Es decir, cómo el museo como agente narrativo nos ha hecho partícipes de unas tramas argumentales sin cuestionar quién ha tenido acceso o ha participado en la construcción de este relato. Al mismo tiempo, dicho ejercicio posibilita un escenario de reflexión en el que el museo, cuestionando dichas estructuras de poder y los privilegios que lo sostienen, se construye -y plantea- otras posibilidades.

La propuesta sitúa la comunicación en un rol central en la búsqueda de significados que nos ayuden a interpretar -y organizar- el presente de manera activa. Supone, además, una (o varias) transgresiones. Mendoza García expone que la acción museística supone «fijar los significados públicos de ciertos símbolos que se pretenden que sean inscritos en la memoria» $(2009,64)$. Es decir, la acción museística es una herramienta de distinción identitaria. En nuestra propuesta, el museo, asumiendo una voluntad de comunicación, ya no establece relaciones verticales con sujetos, sino que propone interacciones con comunidades de prácticas. Las narraciones que emergen de dichas interacciones no solo afectan a los sujetos participantes. La interacción potencia la emergencia de un relato que ayuda a significar la vida cotidiana más allá del relato hegemónico. Dicho relato, producido en la interacción comunicativa entre las comunidades y el museo, construye un imaginario simbólico intersubjetivo, colectivo, negociado y negociable, hegemónico y no hegemónico, pero que visibiliza la memoria colectiva. En este sentido, entendemos la comunicación participativa como una forma de comunicación comunitaria, ya que supone un modelo circular que, en el propio proceso, crea comunidad y, por ende, relato identitario en torno a esta. A su vez, es una acción transformadora: genera la posibilidad de relatos que proponen imaginarios compartidos no hegemónicos. Es

11.Anteriormente a esta investigación, se planteó una IAP en el Museo Marítimo de Barcelona. En esta ocasión se trabajó con mujeres de mar. Todas las participantes, empalabrando la práctica comunitaria, fueron desvelando estrategias para enfrentarse a un sistema patriarcal que las rechazaba por el hecho de ser mujeres. 
https://artnodes.uoc.edu

importante resaltar que no solo supone un proceso de reconocimiento de la voz de los sujetos implicados, sino que estos son escuchados y son parte de la toma de decisiones de los procesos que les afectan como agentes creadores de hibridación.

Los retos de la propuesta son múltiples: establecer qué rol tienen museo y comunidades en una relación que deben ser capaces de construir como horizontal, pero en la que el museo se responsabiliza del espacio que ocupa y de los capitales simbólicos, económicos y culturales que atesora; acoger nuevas formas de participación transversales a las diferentes propuestas y, sobre todo, entender que la propuesta no supone solo un cambio de narrativa: el eje de la propuesta es un cambio de mirada por parte del museo y también por parte de las comunidades, así como de las personas, visitantes potenciales del museo.

\section{Referencias bibliográficas}

Aguado, Juan. M. Comunicación y cognición: bases epistemológicas de la complejidad. Sevilla: Comunicación Social Ediciones y Publicaciones, 2003.

Barrio Fernández, Teresa. El museo como inspirador de valores y armonizador social en tiempos difíciles. Tesis de doctorado. Navarra: Universidad de Navarra, 2017.

Bishop, Claire. Artificial Hells. Participatory Art and the Politics of Spectatorship. Londres y and Nueva York: Verso, 2012.

Bruner, Jerome. La educación, puerta de la cultura, Madrid: Visor, 1997.

Bruner, Jerome. La fábrica de historias. Derechos, literatura, vida. México: Fondo de Cultura Económica, 2003.

Ciudades y Gobiernos Locales Unidos. Carta de Roma 2020. El dret a participar lliurement i plenament a la vida cultural és vital per a les nostres ciutats i comunitats. Roma Capitale y la Comisión de Cultura de CGLU, 2020.

Croizet, Florencia. «Museos inteligentes para superar la crisis: ICOM Voices». ICOM (2021). https://icom.museum/es/news/museos-inteligentes-para-enfrentar-la-crisis/.

Domínguez García, A. La dimensión social de las comunidades marítimas del presente: un análisis a partir de las prácticas cotidianas desde una perspectiva de género y su aplicación a la comunidad pesquera de s'Estanyol de Migjorn. Trabajo de Fin de Máster. Universitat de les Illes Balears (UiB), 2020.

Duch, Lluís. «Antropologia de la comunicació». Anàlisi. Quaderns de Comunicació i Cultura, no. 29 (2002): 21-40.

Duch. Lluís. Sortida del laberint. Barcelona: Fragmenta, 2019.

García Canclini, Néstor. Las culturas populares en el capitalismo. México: Nueva Imagen, 1982.

García Canclini, Néstor. Culturas Híbridas. Buenos Aires: Paidós, 2001.

Garoian, Charles. R. «Performing the museum». Studies in Art Education: A Journal of Issues and Research in Art Education, vol. 42, no. 3 (2001): 234-248. DOl: https://doi.org/10.2307/1321039.
Museos, memoria colectiva e imaginarios narrativos. La comunicación participativa como estrategia para construir relatos no hegemónicos en museos con vocación social

Geertz, Clifford. La interpretación de las culturas. Barcelona: Gedisa, 1987. Gumucio-Dagrón, Alfonso. "Comunicación para el cambio social: clave del desarrollo participativo». Signo y Pensamiento, no. 58 (2011): 26-39.

Hall, Stuart. Representation: Cultural Representations and Signifying Practices. Londres: Sage/The Open University, 1997.

ICOM. «La creación de una nueva definición de museo - la columna vertebral del ICOM». ICOM (2020). https://icom.museum/es/normas-y-directrices/definicion-del-museo/.

Krotz, Esteban. «Alteridad y pregunta antropológica». Alteridades, vol. 4, no. 8 (1994): 5-11. ISSN: 0188-7017.

Loran Gili, M. «La planificació estratégica en museus i equipaments patrimonials». En: 0. López y l. Lorés (eds.) Planificació estratègica de museus i centres patrimonials. (2021): 21-54. Barcelona: Associació de Museòlegs de Catalunya.

Lynch, Enrique. «Discurso interrumpido», Anàlisi, no. 25 (2000): 95-108.

Mercado Maldonado, Asael, Hernández Oliva, Alejandrina V. «El proceso de construcción de la identidad colectiva». Convergencia, vol. 17, no. 53 (2010): 229-251. http://www.scielo.org.mx/scielo.php?script=sci_arttext\&pid=S1405-14352010000200010\&lng=es\&tln$\mathrm{g}=\mathrm{es}$.

Martín Barbero, Jesús. «Comunicación y cultura: unas relaciones complejas». Telos: cuadernos de comunicación, tecnología y sociedad, no. 19 (1989): 21-26.

Mendia, Irantzu, Luxán, Marta, Legarreta, Matxalen, Guzmán, Gloria. Zirion, Iker, Azpiazu, Jokin. Otras formas de (re)conocer. Reflexiones, herramientas y aplicaciones desde la investigación feminista. Bilbao: Hegoa / SIMReF, 2015. http://publicaciones.hegoa.ehu.es/ assets/pdfs/329/0tras_formas_de_reconocer.pdf?1429005444.

Mendoza García, Jorge. «Las formas del recuerdo. La memoria narrativa». Athenea Digital, no. 6 (2004, otoño). Universidad Autónoma de Barcelona. https://www.academia.edu/23464552/Las_formas_del_recuerdo._La_memoria_narrativa. DOl: https://doi. org/10.5565/rev/athenead/v1n6.158.

Moreno Pérez, Arnoldo. «¿Son las ciencias de la comunicación esencialmente incompletas?». La Flecha. Tu diario de ciencia y tecnología (2008). https://laflecha.net/son-las-ciencias-de-la-comunicacion-esencialmente-incompletas/.

Pintos, Juan Luis. Los imaginarios sociales. La nueva construcción de la realidad social. Madrid, Sal Terrae/l, 1995.

Ramiro Beltrán, Luis. «La comunicación para el desarrollo en Latinoamérica: un desencuentro de medio siglo", III Congreso Panamericano de la Comunicación, (2005, 12 -16 julio). Buenos Aires, Argentina.

Rizo García, Marta. «Me comunico, luego existo. El papel de la comunicación en la construcción de identidades». Culturales, vol. I, no. 1 (2005, enero-junio): 124-142. México: Universidad Autónoma de Baja California Mexicali.

Scott, James. C. Los dominados y el arte de la resistencia. México: Edición Era, 2000. 


\section{artnodes}

https://artnodes.uoc.edu

Museos, memoria colectiva e imaginarios narrativos. La comunicación participativa como estrategia para construir relatos no hegemónicos en museos con vocación social

Soliz, Fernanda, Maldonado, Adolfo. Guía de metodologías comunitarias participativas. Ecuador: Universidad Andina Simón Bolívar, 2012. http://repositorio.uasb.edu.ec/bitstream/10644/3997/1/Soliz,\%20 FCON008-Guia5.pdf.

Tresserras, Joan. M. «La cultura popular en els orígens de la cultura de masses». Comunicación y estudios universitarios, no. 6 (1996): 73-80.
Tufte, Thomas. Comunicación para el cambio social. La participación y el empoderamiento como base para el desarrollo mundial. BarceIona: Icaria, 2015.

Wenger, Étienne. Communities of Practice. Learning, Meaning, and Identity. EE. UU.: Cambridge University Press, 1997. D0I: https:// doi.org/10.1017/CB09780511803932.

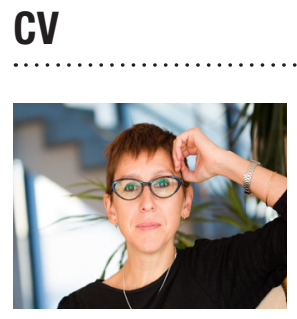

Catalina Gayà Morlà

Universidad Autónoma de Barcelona

Catalina.Gaya@uab.cat

Doctora en Comunicación y profesora lectora Serra Húnter del Departamento de Medios, Comunicación y Cultura (UAB). Es profesora de Políticas culturales en tiempos de capitalismo global, del máster del Departamento y de Escritura Periodística, en el grado. Hasta 2020, fue consultora de la UOC en el máster de Nuevas Narrativas y profesora de Mirada y Método en el máster de Periodismo Literario de la UAB. Ha dirigido el Estudio sobre las percepciones en torno a las violaciones machistas y la igualdad entre los jóvenes de 14 a 18 años de las Islas Baleares; Dona'm la Mar, proyecto del Museo Marítimo de Barcelona en torno a cómo incorporar a las mujeres en sus narrativas, y La remor de la memòria, proyecto participativo comunitario del Museo Marítimo de Mallorca. Dona'm la mar ganó el premio al mejor proyecto otorgado por la Asociación de Museólogos de Cataluña. Tiene más de veinte años de experiencia como periodista en diferentes países, entre ellos México, donde trabajó para la revista Cambio, dirigida por Gabriel García Márquez. «Ve, vive y cuéntalo» -una enseñanza y un regalo del premio Nobel- ha marcado toda su carrera como investigadora de discursos no hegemónicos. Es autora, entre otros, de El mar es tu espejo (2017, Libros del K0) y de Ulises es nombre de mujer. Un relato no oficial del mar (MMB-Diputación de Barcelona, 2021).

https://orcid.org/0000-0001-6190-6824 


\section{artnodes}

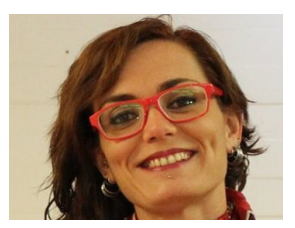

\section{Marta Rizo García}

Universidad Autónoma de la Ciudad de México y Universidad Autónoma de Barcelona

mrizog@gmail.com

Licenciada, máster y doctora en Comunicación por la Universidad Autónoma de Barcelona. De 2001 a 2002 fue investigadora del Laboratorio de Comunicación Compleja (LabComplex) en la Universidad Iberoamericana de México. Desde 2003 es profesora titular de la Academia de Comunicación y Cultura de la Universidad Autónoma de la Ciudad de México, y desde 2020 es profesora colaboradora de la maestría en Innovación Comunicativa para las Organizaciones de la Universidad Autónoma de San Luis Potosí (México) y del grado de Comunicación y el máster en Periodismo y Comunicación Digital de la Universitat Oberta de Catalunya (España). A partir de febrero de 2022 es profesora lectora en el Departamento de Medios, Comunicación y Cultura de la Universidad Autónoma de Barcelona. De 2008 a 2020 coordinó el grupo de investigación Comunicación Intersubjetiva de la Asociación Mexicana de Investigadores de la Comunicación, y desde 2018 es vicecoordinadora del grupo de trabajo Teoría y metodología de la investigación en comunicación de la Asociación Latinoamericana de Investigadores de la Comunicación. Es autora de 19 libros, más de 100 artículos académicos, y más de 60 capítulos de libro. Ha sido ponente y conferencista en cerca de 200 eventos en México, España, Portugal, Alemania, Francia, Cuba, Panamá, Perú, Argentina, Bolivia, Uruguay, Brasil, Costa Rica y Colombia, entre otros países. Es miembro del Sistema Nacional de Investigadores (Nivel II) del Consejo Nacional de Ciencia y Tecnología (México). Integra el Comité Editorial de más de 20 revistas académicas de México, Brasil, Chile, Argentina y España, entre otros países.

https://orcid.org/0000-0003-3066-1419

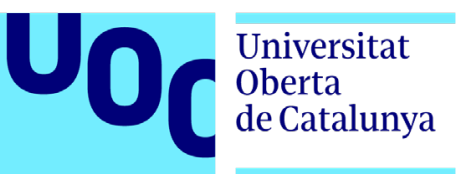

